\title{
Inadvertent Migration of Umbilical Venous Catheters Often Leads to Malposition
}

\author{
Gerdina H. Dubbink-Verheij ${ }^{a}$ Remco Visser ${ }^{a}$ Ratna N.G.B. Tan ${ }^{a}$ \\ Arno A.W. Roest ${ }^{b}$ Enrico Lopriore ${ }^{a}$ Arjan B. te Pas $^{a}$ \\ a Division of Neonatology, Department of Pediatrics, Leiden University Medical Center, Leiden, The Netherlands; \\ ${ }^{\text {b}}$ Division of Cardiology, Department of Pediatrics, Leiden University Medical Center, Leiden, The Netherlands
}

\section{Keywords}

Umbilical venous catheters · Migration · Malposition

\begin{abstract}
Background: Migration of umbilical venous catheters (UVCs) has been described anecdotally. Objectives: The aim of this paper was to investigate migration of UVCs using ultrasonography (US). Methods: In a prospective observational study, the position of UVCs was determined using serial US within 24 h, at midweek, and at the end of the week after umbilical catheterization. Migration was recorded in distance and direction. Malposition was defined as a position of the UVC in the heart (right atrium or more distal along the UVC-route), umbilicoportal confluence, or in the umbilical vein. UVC position determined by US was compared with chest X-rays (CXRs) when these were performed for standard care within the same period of $1 \mathrm{~h}$. Results: Migration of UVCs was detected with US in $25 / 40$ infants (63\%) in 32 occasions, leading to malposition in 17/25 (68\%) infants. UVCs migrated inwards in 18/32 (56\%), leading to a position within the heart in 17/18 occasions. Most migrations occurred before Day 3 (21/32 [66\%]). When a CXR was taken at the same time as US was performed (30 occasions), the assess-
\end{abstract}

\begin{tabular}{ll}
\hline KARGER & $\begin{array}{l}\text { ( } 2019 \text { The Author(s) Karger } \\
\text { Published by S. Karger AG, Basel Open caccess }\end{array}$ \\
E-Mail karger@karger.com & $\begin{array}{l}\text { This is an Open Access article licensed under the Creative Commons } \\
\text { Attribution-NonCommercial-4.0 International License (CC BY-NC) } \\
\text { (http://www.karger.com/Services/OpenAccessLicense), applicable to } \\
\text { the online version of the article only. Usage and distribution for } \\
\text { commercial purposes requires written permission. }\end{array}$
\end{tabular}

ment of the catheter-tip position differed in $23 \%$ of the occasions. When malposition was detected by US, this was detected on routinely performed CXRs in $11 \%$ of the occasions. Conclusions: UVCs often migrate following insertion, often leading to malposition. Awareness for this is needed, and US is a feasible alternative for detecting malposition compared to CXRs and avoids additional radiation. Re-evaluation of the position of UVCs at least once, but within $24 \mathrm{~h}$ after placement, is recommended.

(C) 2019 The Author(s) Published by S. Karger AG, Basel

\section{Introduction}

Umbilical venous catheters (UVCs) are frequently used in sick infants for intravenous access. UVCs are relatively easy to insert and may be used for a longer period in comparison to peripheral intravenous cannulas. Catheters are introduced in the umbilical vein to reach the umbilicoportal confluence, passing the ductus venosus to reach the junction between the inferior vena cava and right atrium (IVC-RA junction) (Fig. 1). The ideal location for the UVC tip is between the insertion of the ductus 


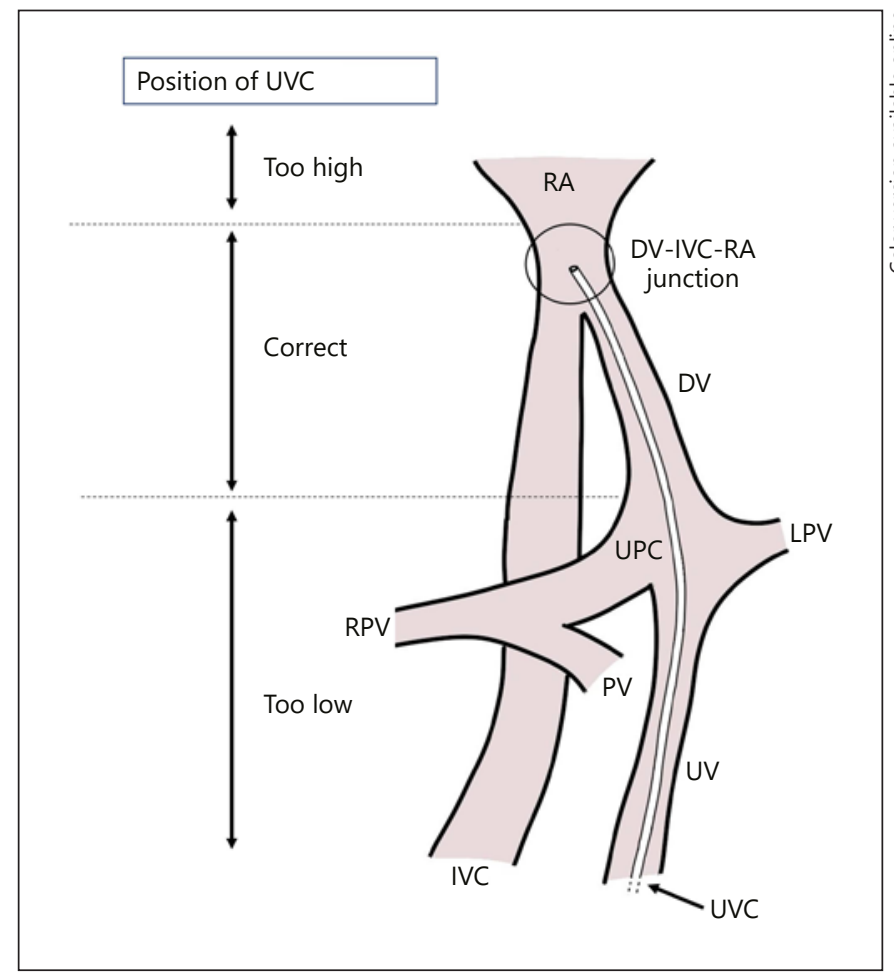

Fig. 1. Relevant anatomy for the UVC route with too high, correct, and too low locations for the UVC indicated. RA, right atrium; DV, ductus venosus; UPC, umbilicoportal confluence; LPV, left portal vein; RPV, right portal vein; PV, portal vein; IVC, inferior vena cava; UV, umbilical vein; UVC, umbilical venous catheter.

venosus in the IVC and the IVC-RA junction [1-4]. It is important to prevent malposition as this may lead to complications, such as hepatic necrosis, thrombosis, and cardiac arrhythmias $[1,4-10]$.

Migration of UVCs was regularly observed in our neonatal intensive care unit (NICU) on chest X-rays (CXRs) in the following days after placement, despite fixation of the catheter with stitches in the umbilical cord and adhesive plasters to the abdominal wall. Although there are several reports on the placement and location of UVCs, there is little data on the occurrence of migration after placement, and how often this leads to malposition. Gupta et al. reported that on CXR $46 \%$ of UVCs migrate into the cardiothymic silhouette requiring repositioning within $24 \mathrm{~h}$ after placement [11]. Although CXR is still a commonly used imaging modality for determination of UVC position, it is not a very accurate method to estimate the location of the UVC and migration leading to malposition can be overlooked [12]. Ultrasonography (US) is more accurate and increasingly advocated for this purpose [13].
The aim of our study was to describe the migration of UVCs using US.

\section{Methods}

\section{Study Design and Patient Population}

We conducted a prospective observational study at the NICU of the Leiden University Medical Center from October 1, 2016, until October 1, 2017. All infants who had a UVC inserted were eligible for the study.

\section{Umbilical Catheterization and Assessment of UVC Position}

According to our protocol, UVCs were placed in all neonates born at a gestational age $<28$ weeks or with a birth weight $<1,000$ $\mathrm{g}$ and in all other neonates that needed mechanical ventilation or circulatory support by inotropes. Other indications for a UVC at our unit are difficulties to get intravenous access and the need for an exchange transfusion. To determine the depth of the UVCs, the revised formula of Shukla was used [14]. The technique used for umbilical catheterization is described and demonstrated by Anderson et al. [5], aiming for the position of the UVC tip at the IVCRA junction. The UVC (4-, 5-, or 8-French catheter; Vygon, France) was sutured in place to the umbilical cord and taped to the infant's abdomen. An anteroposterior CXR was routinely performed to determine the position of the UVC directly after catheterization.

Serial US was performed at the day of placement of the UVC (Day 1), during midweek, and at the end of week 1 after placement (or sooner when the UVC was removed). The exact day depended on the presence of a caregiver skilled in US. Furthermore, in case of a catheter dwell time of $>7$ days, an extra US was performed on the day of removal. When CXR was also performed on the day an US was planned, US was performed within the same period of $1 \mathrm{~h}$.

The position of the catheter tip was determined by US, and the distance between the catheter tip and the IVC-RA junction was measured. The ultrasonographist was blinded to the result of the CXR at the time of performing US. US was performed by skilled neonatologists or pediatric cardiologists. The correct position of the UVC on the ultrasound scan was defined as a UVC with the catheter tip in the ductus venosus or at the IVC-RA junction $[3,4]$ (Fig. 1). Malposition was defined as the catheter tip in the heart, in the umbilicoportal confluence, or in the umbilical vein. The position was defined too high when the UVC tip was in the heart (RA or more distal along the UVC route). The position was defined too low when the UVC tip was in the umbilicoportal confluence or umbilical vein. Infants with UVCs positioned too low or UVCs with the catheter tip in branches of the portal vein were excluded, and the UVC was removed within $1 \mathrm{~h}$ after insertion.

The correct position of the UVC on CXR according to the vertebral level method was defined as a UVC with the catheter tip projecting on the ninth or tenth thoracic vertebral level. The position was defined too low when the catheter tip projected below the tenth thoracic vertebral level and too high projecting above the ninth thoracic vertebral level. One of the researchers (G.H.D.-V.) reviewed all CXRs.

US was performed with a Toshiba Aplio 400 or Aplio i700 machine (Toshiba Medical Systems Europe B.V., The Netherlands) with multi-frequency transducers. Standard two-dimensional 
gray-scale ultrasound images were acquired from at least subcostal, abdominal, and apical four-chamber views, with parasternal short-axis views as needed, and were stored in digital format. To achieve better visualization of the exact position of the catheter tip, a saline solution ( $\max 1 \mathrm{~mL}$ ) was injected in the catheter as a contrast medium when deemed necessary.

When the UVC tip was too high, the UVC was repositioned directly after CXR or US if deemed necessary. All repositioning actions, including their distance, were recorded. Standard repeat examinations to document the final position of the UVC after repositioning were not made.

Migration of the UVC on US was defined by one of the following two criteria: criterion 1 was fulfilled when the catheter tip was positioned in another anatomical structure than on prior US or than expected after repositioning. Criterion 2 was fulfilled when the distance between the catheter tip and the IVC-RA junction differed $>0.5 \mathrm{~cm}$ from this distance on prior US or $>0.5 \mathrm{~cm}$ from the expected distance after repositioning.

\section{Outcome Measures}

The primary outcome was the proportion of migration of UVCs after placement detected on US. Secondary outcomes were the time point when migration was detected, the proportion of malposition, and the location. We also compared the detection of migration and malposition of UVCs by US with CXRs when one was taken.

\section{Data Collection and Statistical Analysis}

Data collected included gestational age, birth weight, mode of respiratory support, weight at subsequent ultrasound scans, date and time of UVC placement and removal, timing of all CXRs and ultrasound scans, position of the catheter tip on CXR and US, and inward and outward migration. The baseline characteristics of each infant and clinical data needed were retrieved from the electronic patient dossier Metavision (iMD-soft, Leiden, The Netherlands).

Data are given as median with interquartile range (IQR) or range for continuous data, and number (percentage) for dichotomous data where appropriate. The $\chi^{2}$ test was used to compare the incidence of migration in the different periods and groups, and the Kruskal-Wallis test was employed in case of more than two groups. $p$ values $<0.05$ were considered statistically significant. Statistical analyses were performed with IBM SPSS Statistics version 23.0 (IBM Software, Armonk, NY, USA).

\section{Results}

During the study period, umbilical catheterization was performed in 143/629 infants, of whom 103 were excluded because consent could not be obtained or a skilled ultrasonographist was not available. In total, 40 infants were included with a median gestational age of 27 weeks (range 24-41) and a birth weight of 1,053 g (range 600$3,925)$. Respiratory support was given in 36/40 (90\%) infants, of whom 12/40 (30\%) received mechanical ventilation and 24/40 (60\%) received continuous positive airway

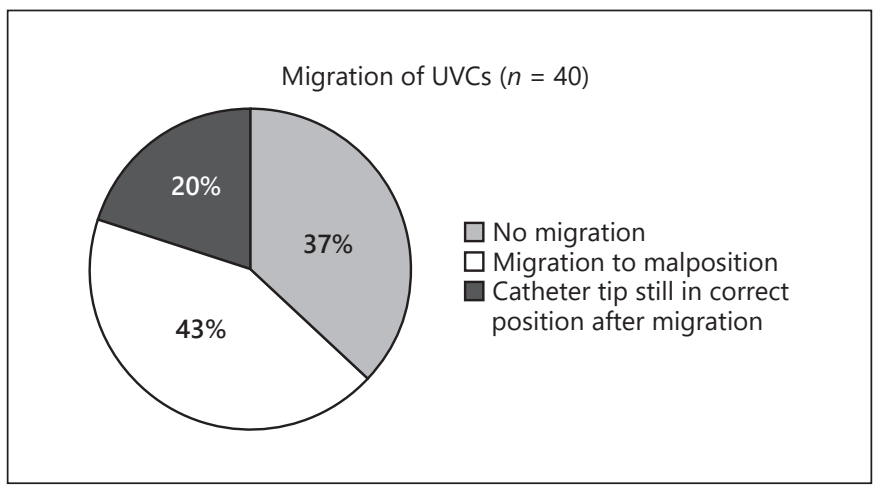

Fig. 2. Migration of umbilical venous catheters in 40 infants.

pressure at the moment of catheterization. UVCs were placed in 30 infants on the day of birth (Day 1), in 9 infants on Day 2, and in 1 on Day 3 after birth. The median dwelling time of the UVC was 7 days (IQR 5-8). US was performed on Day 1 and on median Day 3 (IQR 3-3), 7 (IQR 6-7) and 8 (IQR 8-10).

\section{Migration}

Serial US detected migration in 25/40 (63\%) infants. In $17 / 25$ (68\%) infants, this led to malposition (Fig. 2). Migration occurred twice in 7 infants, leading to 32 migrations in 25 infants. In 7/32 (22\%) occasions, criterion 1 of migration was fulfilled (the UVC tip migrated to another anatomical structure), in 5/32 (16\%) occasions, criterion 2 was fulfilled (the UVC tip migrated over a distance $>0.5$ $\mathrm{cm}$ ), and in 20/32 (63\%) occasions, both criteria were fulfilled. In 15/32 (47\%) occasions, the UVC tip even moved over a distance $>1 \mathrm{~cm}$. In 18/32 (56\%) occasions, UVCs migrated inwards, which led to the catheter tip in the heart in $17 / 18$ (94\%) occasions. In 14/32 (44\%) occasions, UVCs migrated outwards, which led to a catheter tip that was too low in 2/14 (14\%) occasions. In 19 occasions with migration detected by US (in 17 infants), the UVC was migrated to an incorrect position, whereas 2 out of these 19 occasions (11\%) were detected by routinely performed CXRs.

In 38 infants, the first US was performed within the first $24 \mathrm{~h}$ after UVC placement, in 21/38 infants within $1 \mathrm{~h}$ after placement, and in 17/38 infants $9 \mathrm{~h}$ (median) after placement (IQR 3-14 h). The first US showed that the catheter tip was in a correct position in 5/38 (13\%) infants and positioned too high in $32 / 38$ infants ( $84 \%)$. Five out of 32 UVCs positioned too high were accepted by the caregiver without repositioning (protocol not followed). The other 27/32 UVCs that were positioned too high were 
Table 1. Migration of umbilical venous catheters

\begin{tabular}{llll}
\hline & $\begin{array}{l}\text { Day 3 } \\
(n=37)\end{array}$ & $\begin{array}{l}\text { Day 7 } \\
(n=24)\end{array}$ & $\begin{array}{l}\text { Day 8 } \\
(n=11)\end{array}$ \\
\hline $\begin{array}{l}\text { No migration } \\
\text { Migration }\end{array}$ & $16(43)$ & $15(63)$ & $9(82)$ \\
$\quad$ Inward, too high & $21(57)$ & $9(38)$ & $2(18)$ \\
$\quad$ Inward, still correct & 11 & 5 & 1 \\
$\quad$ Outward, too low & 2 & - & - \\
Outward, still correct & 7 & 4 & - \\
\hline
\end{tabular}

Data are represented as number (percentage). Time expressed in days of umbilical venous catheterization. Day 1: insertion day, Day 3 (IQR 3-3), Day 7 (IQR 6-7), Day 8 (IQR 8-10).

Table 2. Chest X-ray (CXR) versus ultrasonography (US) for the position of the umbilical venous catheter tip

\begin{tabular}{llll}
\hline & $\begin{array}{l}\text { Malposition } \\
\text { on US }\end{array}$ & $\begin{array}{l}\text { Correct } \\
\text { position } \\
\text { on US }\end{array}$ & Total \\
\hline Malposition on CXR & 19 & 5 & 24 \\
Correct position on CXR & 2 & 4 & 6 \\
\hline Total & 21 & 9 & 30 \\
\hline
\end{tabular}

Data are represented as numbers.

repositioned directly after US. In 1 infant, the exact position of the UVC tip could not be determined due to limited visualization by US. In the infants with US performed within $1 \mathrm{~h}$ after placement, the incidence of migration on the following ultrasound scan was higher (17/21 [81\%]) than in the infants with the first US performed later (median $9 \mathrm{~h})$ after UVC placement $(7 / 17$ [41\%]) $(p=0.01)$. The occurrence of migration observed during midweek and the end of the week was not different $(p=0.06)$, although there was a trend towards a higher migration rate during midweek (Table 1).

In 30 occasions, a CXR and US was performed within the same period. Positioning was different when assessed with CXR compared to US in 7/30 (23\%) occasions, and 2/21 (10\%) malpositions were missed on CXR (Table 2).

In 6/40 infants, intracardiac thrombosis was detected by US. In 4 of these infants, inward migration of the UVC was observed. None of these 4 infants needed antithrombotic treatment. No cardiac arrhythmias were observed in the study population.

\section{Discussion}

In this prospective study, we observed that UVCs often migrate $(63 \%)$ in the days after placement, despite securing them in the correct position. This often led to malpositions of the catheter. Most UVCs migrated inwards to a position in the heart, which is potentially dangerous. Outward migration was also seen, but in most of these cases, the migrated UVC was still in an acceptable position. These findings imply that caregivers should be more aware of the possibility of catheter migration, and the position should be rechecked in the days after placement.

Migration of UVCs has been reported anecdotally [15-17]. Case reports described UVC-related complications such as pericardial effusion, left atrial thrombosis, and intrahepatic hematoma in infants with a migrated UVC at the time of detection of the complication $[15,16]$. Gupta et al. [11] reported that $36 \%$ of the UVCs migrated into the cardiothymic silhouette on CXRs within $1 \mathrm{~h}$ after insertion, and $23 \%$ after $24 \mathrm{~h}$. Franta et al. [18] very recently performed a prospective study using serial US. During the inclusion period of our study, they reported a migration incidence of $50 \%$ and, despite adjustments of the UVC position if needed, malposition re-occurred in $23-62 \%$ on the different scans. Catheter migration in this study was defined as a change in the catheter location from the optimal position to malposition or from one malpositioned site to another. In our study, we assessed a UVC with the catheter tip still in the correct position as migrated as well if it migrated to another anatomical structure or over a distance of at least $0.5 \mathrm{~cm}$. Only including migration of UVCs that result in malpositions, our migration rate would be $42 \%$, thus comparable to the migration rate in the study of Franta et al. [18].

Although we calculated the insertion length of the UVC by a revised formula of Shukla to avoid positioning that is too high [14], the $13 \%$ correctly positioned UVCs in our population was disappointing. Studies, including previous studies at our department and studies using the same formula, reported variable success in correct positions (14-56\%) [14, 19-23]. Caregivers probably prefer (intentionally or not) to insert UVCs rather too high than too low, thus adding some length to the calculated length. This may contribute to the large proportion of UVCs positioned too high in our and other studies.

In all above-mentioned studies, the position of the catheter tip is determined by interpreting CXRs. There is limited data demonstrating that US is superior for determining the position of the UVC when compared to CXR $[10,12,13,18,24,25]$. Although other authors report 
poor correlation between the thoracic level by CXR and catheter location by US [10,12, 13, 18, 24, 25], we found agreement between CXR and US in 23/30 imaging pairs. However, comparison of US and CXR in determining the UVC position was not the primary goal of this study, and conclusions about the accuracy of both modalities cannot be drawn. Moreover, to limit the burden of radiation to the infant, CXRs are not serially performed at our NICU. During the study period, only 2 out of 19 occasions with malposition due to migration were detected by routinely performed CXRs.

To prevent migration of UVCs and possible subsequent complications of malpositions, knowledge of causes and risk factors for this phenomenon is critical. Abdominal girth variation is suggested as a possible cause of UVC migration $[15,18]$. Some authors speculate that abdominal distension will lead to outward migration [18]. Following this theory, weight loss of the infant could lead to inward migration due to a smaller abdominal diameter. Others explain inward migration of the UVC by a downward movement of the IVC-RA junction caused by lung expansion and diaphragmatic descent, resulting in an upward movement of the catheter tip [16]. Other risk factors for migration have been suggested, such as birth weight, weight and postnatal age at insertion, and changes in the ventilation mode $[11,18]$. Although we documented ventilation mode and weight loss, our study group was too small to investigate the relation of these factors with UVC migration.

We did not perform a standard CXR or US to confirm a correct position of the UVC after repositioning. However, in cases of repositioning after US, subsequent migration of the UVC was assessed based on the expected location of the UVC tip after pulling back the catheter. For repositioning, we measured the distance between the UVC tip and IVC-RA junction and recorded the distance and direction of all repositioning actions.

The high proportion of inward migration in our study is concerning. The location of the UVC tip in the heart is associated with life-threatening complications, such as cardiac arrhythmias, pericardial effusion, and cardiac tamponade [4]. In only $1 / 40$ infants we could not visualize the UVC tip by US. To improve the detection of migration and malposition of UVCs, US is a valuable and feasible tool, as it mostly takes only a few minutes and avoids additional exposure to radiation $[17,24]$. Our study showed that inadvertent migration occurs frequently between the placement of the UVC and Day 3. We speculate that most migration takes place during the first hours after placement, as migration (after initial US and repositioning, if needed) was observed more frequently in infants with the initial US performed within $1 \mathrm{~h}$ of the first CXR (thus, shortly after catheterization) compared to infants with initial US performed later (median $9 \mathrm{~h}$ ). It is possible that we underestimated the occurrence of migration, as this could have been missed when US was not performed within $1 \mathrm{~h}$. It is likely that performing only one US $24 \mathrm{~h}$ after the initial placement could detect most migrations and thus prevent complications.

In conclusion, UVCs frequently migrate inadvertently during the first hours and days following insertion. Part of the UVCs migrate to malposition, especially in the heart. We recommend re-evaluating the position of the catheter at least at one time point, but within $24 \mathrm{~h}$ after placement. This could be done by repeating CXR. However, to avoid additional radiation, US would be preferred. Incorporating US in daily practice at the NICU will detect migration of UVCs better and possibly prevent subsequent complications.

\section{Acknowledgement}

We would like to thank Hans Dubbink for his valuable support in manufacturing Figure 1 of this article.

\section{Statement of Ethics}

The study was approved by the hospital institutional review board. Written informed consent was obtained from the parents of the participating infants.

\section{Disclosure Statement}

The authors have no conflicts of interest or funding to disclose.

References

1 Hogan MJ. Neonatal vascular catheters and their complications. Radiol Clin North Am. 1999 Nov;37(6):1109-25.

2 Rand T, Kirchner L, Puig S, Ponhold W, Vergesslich $\mathrm{K}$, Imhof $\mathrm{H}$. ["Lines and tubes" in neonatal intensive care patients]. Radiologe. 2000 Jan;40(1):52-7.

3 Simanovsky N, Ofek-Shlomai N, Rozovsky K, Ergaz-Shaltiel Z, Hiller N, Bar-Oz B. Umbilical venous catheter position: evaluation by ultrasound. Eur Radiol. 2011 Sep;21(9):1882-6.

4 Hermansen MC, Hermansen MG. Intravascular catheter complications in the neonatal intensive care unit [vii.]. Clin Perinatol. 2005 Mar;32(1):141-56. 
5 Anderson J, Leonard D, Braner DA, Lai S, Tegtmeyer K. Videos in clinical medicine. Umbilical vascular catheterization. N Engl J Med. 2008 Oct;359(15):e18.

6 Ahluwalia JS, Kelsall JL. A. Procedures and iatrogenic disorders. In: Jm R, editor. Roberton's Textbook of Neonatology. Philadelphia, USA: Elsevier; 2008. pp. 1237-72.

7 Bradshaw WT, Furdon SA. A nurse's guide to early detection of umbilical venous catheter complications in infants. Adv Neonatal Care. 2006 Jun;6(3):127-38.

8 Korver AM, Walther FJ, van der Molen AJ, de Beaufort AJ. [Serious complications of umbilical venous catheterisation]. Ned Tijdschr Geneeskd. 2007 Oct;151(40):2219-23.

9 Verheij G, Smits-Wintjens V, Rozendaal L, Blom N, Walther F, Lopriore E. Cardiac arrhythmias associated with umbilical venous catheterisation in neonates. BMJ Case Rep. 2009;2009:bcr04.2009.1778.

10 Raval NC, Gonzalez E, Bhat AM, Pearlman SA, Stefano JL. Umbilical venous catheters: evaluation of radiographs to determine position and associated complications of malpositioned umbilical venous catheters. Am J Perinatol. 1995 May;12(3):201-4.

11 Gupta R, Drendel AL, Hoffmann RG, Quijano CV, Uhing MR. Migration of Central Venous Catheters in Neonates: A Radiographic Assessment. Am J Perinatol. 2016 May;33(6): $600-4$.
12 Michel F, Brevaut-Malaty V, Pasquali R, Thomachot L, Vialet R, Hassid S, et al. Comparison of ultrasound and X-ray in determining the position of umbilical venous catheters. Resuscitation. 2012 Jun;83(6):705-9.

13 Sharma D, Farahbakhsh N, Tabatabaii SA. Role of ultrasound for central catheter tip localization in neonates: a review of the current evidence. J Matern Fetal Neonatal Med. 2018 Feb:1-9.

14 Verheij GH, te Pas AB, Smits-Wintjens VE, Šràmek $\mathrm{A}$, Walther FJ, Lopriore $\mathrm{E}$. Revised formula to determine the insertion length of umbilical vein catheters. Eur J Pediatr. 2013 Aug;172(8):1011-5.

15 Salvadori S, Piva D, Filippone M. Umbilical venous line displacement as a consequence of abdominal girth variation. J Pediatr. 2002 Nov;141(5):737.

16 Abiramalatha T, Kumar M, Shabeer MP, Thomas N. Advantages of being diligent: lessons learnt from umbilical venous catheterisation in neonates. BMJ Case Rep. 2016 Feb; 2016:bcr2015214073.

17 Hoellering AB, Koorts PJ, Cartwright DW, Davies MW. Determination of umbilical venous catheter tip position with radiograph. Pediatr Crit Care Med. 2014 Jan;15(1):56-61.

18 Franta J, Harabor A, Soraisham AS. Ultrasound assessment of umbilical venous catheter migration in preterm infants: a prospective study. Arch Dis Child Fetal Neonatal Ed. 2017 May;102(3):F251-5.

19 Finn D, Kinoshita H, Livingstone V, Dempsey EM. Optimal Line and Tube Placement in Very Preterm Neonates: An Audit of Practice. Children (Basel). 2017 Nov;4(11):E99.
20 Mutlu M, Parıltan BK, Aslan Y, Eyüpoğlu İ, Kader Ş, Aktürk FA. Comparison of methods and formulas used in umbilical venous catheter placement. Turk Pediatri Ars. 2017 Mar; 52(1):35-42.

21 Dongara AR, Patel DV, Nimbalkar SM, Potana N, Nimbalkar AS. Umbilical Venous Catheter Versus Peripherally Inserted Central Catheter in Neonates: A Randomized Controlled Trial. J Trop Pediatr. 2017 Oct;63(5): 374-9.

22 Lean WL, Dawson JA, Davis PG, Theda C, Thio M. Accuracy of five formulae to determine the insertion length of umbilical venous catheters. Arch Dis Child Fetal Neonatal Ed. 2018 Mar;fetalneonatal-2017-314280.

23 Verheij GH, Te Pas AB, Witlox RS, SmitsWintjens VE, Walther FJ, Lopriore E. Poor accuracy of methods currently used to determine umbilical catheter insertion length. Int J Pediatr. 2010;2010:873167.

24 Karber BC, Nielsen JC, Balsam D, Messina C, Davidson D. Optimal radiologic position of an umbilical venous catheter tip as determined by echocardiography in very low birth weight newborns. J Neonatal Perinatal Med. 2017;10(1):55-61.

25 Harabor A, Soraisham A. Rates of intracardiac umbilical venous catheter placement in neonates. J Ultrasound Med. 2014 Sep;33(9): 1557-61. 